\title{
Use of biological mesh in trans-anal treatment for recurrent recto-urethral fistula
}

\author{
Marco Inama ${ }^{1}$ (1)
}

Received: 7 September 2017 / Accepted: 11 September 2017 / Published online: 16 September 2017

(C) Springer Science+Business Media B.V. 2017

Dear Editor,

I read with interest the letter by Deepak Batura about our paper entitled "Use of biological mesh in trans-anal treatment for recurrent recto-urethral fistula". I thank the colleague for his accurate comments. I take this opportunity to clarify the following points:

1. All 7 patients reported in the original article underwent double diversion at least 2 months before the author's procedure. In this situation, no enteric preparation is necessary with a surgical field clean and ideal to use a mesh in a potential infected site. The biological mesh has a great capacity to progressively integrate itself;

2. All 7 patients we described underwent extensive clinical investigations during serial follow-up visits that excluded urethral stricture, signs of infections or urethral sequelae. That is way the patients did not undergo extra examinations (endoscopic or bacteriological) once the stoma was closed.

I hope this additional information will help to clarify your queries.

\section{Compliance with ethical standards}

Conflict of interest None.

Marco Inama

inama.marco@gmail.com

1 General Surgery Unit, Hospital "Dott. Pederzoli”, Via Monte Baldo 24, Peschiera del Garda, 37019 Verona, Italy 